\title{
Undergraduate psychiatry in Hong Kong
}

\section{An elective experience}

\author{
Duke Nguyen
}

\begin{abstract}
This is a report of a four week elective experience in the poychiatiry department of the Chinese University of Hong Kong. Psychlatry is still a growing field in Hong Kong and the general attliudes to psychiatiy are not dissimilar to those seen in the UK.
\end{abstract}

During my time in Hong Kong in 1994, I actively participated in the psychiatry department and followed the fourth year medical student psychiatry rotation. I attended ward rounds, out-patient clinics and had close contact with the teaching staff and senior members of the department.

Hong Kong is a small British colony with a dense population of 6 million: $98 \%$ are predominantly Cantonese-speaking Chinese who live under both Chinese and Western cultural influences. In recent years, the country has undergone fast economic growth with the second highest GDP per capita (US\$18364 in 1993) in the Far East (Government Information Offices, 1993).

There are two medical schools in Hong Kong: The University of Hong Kong (established 1912) and the Chinese University of Hong Kong (established 1963, first medical student intake 1981). Both have adopted the British system of medical teaching and use English as the teaching medium. The high standard of training has meant that graduates from these medical schools are recognised by the General Medical Council (GMC) for the purpose of registration to practise in the UK.

The Chinese University of Hong Kong (CUHK) medical students are exposed to psychiatry from the first year. The module is the joint responsibility of the psychiatry, and the community and family medicine departments, and includes a series of lectures in psychology and sociology illustrated with clinical examples. The students participate in two practical exercises. In the family follow-up project students follow a newborn infant from birth to the age of four and report on the physical and emotional growth of the infant with respect to the psycho-environment of the family. In the psychiatric interview practicum students interview a psychiatric inpatient from the teaching hospital and discuss their findings; their interviewing technique is also reviewed. This early exposure to clinical work may stimulate thought of a future career in psychiatry but also allows students to discover their capabilities and limitations, which is advantageous for other aspects of clinical medicine.

In the second clinical year, students attend a series of psychiatry seminars and undergo a ten week clinical psychiatry rotation. In addition to the written and clinical examinations at the end of the rotation, students are tested at the end of the year in the combined clinical examination by representatives of the four departments in charge of the second clinical year teaching, i.e. paediatrics, obstetrics and gynaecology, community and family medicine, and psychiatry departments. The patient may belong to any of the four specialities and both physical and psychosocial aspects are assessed. This is on a par with the curriculum in many British medical schools, an impressive feat considering psychiatry is still a developing field in Hong Kong.

Psychiatry as a career was not at the top of a medical student's list, similar to the attitudes of most British students. A small number of students did however, even at this early stage, show a high degree of interest in the subject. The ideas of total patient care and long-term follow-up were attractive. Although some students felt there may be an overuse of medication in certain psychiatric conditions such as neurotic illness and preferred to explore the patient holistically. they did at the same time recognise that their medical training would not become obsolete as some of their peers suggested, acknowledging that psychiatry is not just a talking speciality but also requires the complementary use of medicine. Those who were thinking about psychiatry as a career showed the ability to tolerate ambiguity, suggesting it was a misconception which may have arisen due to the many possibilities in the speciality. The students enjoyed the human contact and communication with patients, not simply arriving at the diagnosis and drawing up a 
management plan. They showed a high degree of curiosity towards other individuals' emotions, believing that by learning about other people's psyche, they would benefit both professionally and in their personal lives. Many developed an interest in the fleld after starting the rotation, supporting the observation that a longer clerkship and quality teaching do stimulate greater interest in medical students to becoming psychiatrists.

Interestingly, all students stated that they enjoyed the psychiatry rotation, although not necessarily for the right reasons: a number of students welcomed the ten weeks because it gave them the opportunity to catch up on the thousands of pages of text they felt required to read during their other specialities!

Until this year, when the Concise Oxford Text book of Psychiatry was published, it was difficult to convince students not to use the Oxford Textbook of Psychiatry. Many felt that Essential Psychiatry and other similar sized books were too small'. The standard of the students' English was good but lack of practice hindered their history presenting techniques. I wonder whether this status will remain when Britain relinquishes its sovereignty in 1997, and ties with the GMC will almost certainly be severed. In lectures and case conferences students tended to keep quiet until 'summoned' to express their views. Chinese culture encourages collectivism i.e. individuals only identify themselves as part of a group; the group is invariably their immediate family. The hierarchy of the family is revered, the views of the elders are always respected. Moreover, children are taught to respect their teachers as they would their parents. Thus, students were generally very compliant and rarely challenged their teachers' views. It has been suggested that Chinese patients tend to somatise and show little emotion (Cheng, 1989). However, my impression was that they showed emotions readily.

The elective experience has been of great benefit at a personal level. I believe that the more cultures one is exposed to the more one is tolerant of another's view so opinions can become more informed and balanced.

\section{Acknowledgements}

I am grateful to the senior members of the Psychiatry department of the Chinese University of Hong Kong, Prof. C. N. Chen, Drs. C. K. Wong and S. Lee and Prof. A. H. Crisp, department of Psychiatry, St. George's Hospital Medical School, London, for their help and advice.

\section{References}

GOVERNMENT INFORMATION OFFTCES (1993) Hong Kong 1994: A review of 1993. (ed. R. Daryananil): Hong Kong: Government of Hong Kong.

ChEnG, T. A. (1989) Symptomatology of minor psychiatric morbidity: a cross culture comparison. Psychological Medicine, 19, 697-708.

Duke Nguyen, Final year medical student, St. George's Hospital Medical School, Cranmer Terrace, London SW17 ORE 